\title{
Role of Artificial Neural Network in Welding Technology: A Survey
}

\author{
Amit Kumar, Vikas Chauhan, Ankur Singh Bist \\ G.B. Pant University of Agriculture \&Technology, Pantnagar, India
}

\begin{abstract}
This paper a survey of artificial neural network on various welding technology and also discusses the result based on analysis. The role of optimization techniques in concerned domain with experimental analysis is explained.
\end{abstract}

\section{INTRODUCTION}

There are many mathematical models by which we can control the quality of weld properties in arc welding. Neural network is used where some relationship between input and output. Now a days neural network is very useful tool by which input parameter and final output can be interrelated and compare it with that of the value which is given by the neural network and can optimize the value. An artificial neural network and genetic algorithm is use the optimize the parameter. An Artificial Neural Network is a mathematical model inspired by biological neural networks. which shows some kind of relationship between input and output.

\section{Neural Network:-}

A neural network consists of an interconnected group of artificial neurons, and it is very important and useful tool for develop models which express the interrelationship between input data and output data. There are so many advantages of ANN in the engineering design and GT(group technology) because it can store a large set of parameter In most cases a neural network is an adaptive system that mutates its structure during a learning phase. Learning of Neural network is very important phase of it. Neural networks are crucial to model complex relationships between inputs and outputs or to find patterns in data.

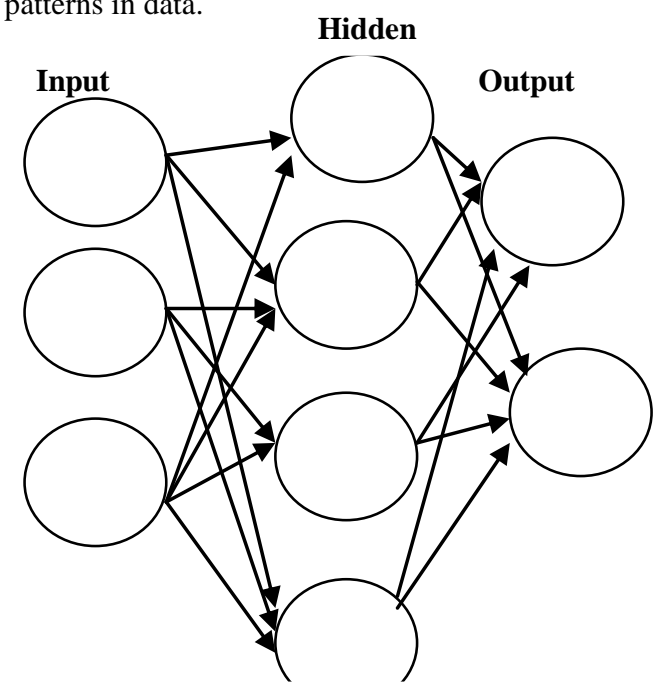

Figure 1. Neural Network
Neural networks are similar to biological neural networks in that functions are performed collectively and in parallel by the units it is very important point because the biological neurons are perform the function in parallel artificial neural network are not work accurately like biological neuron, rather than there being a clear delineation of tasks in smaller groups to which various units are assigned. The term "neural network" usually refers to models employed in statistics, cognitive psychology and artificial intelligence. Neural network models which emulate the central nervous system are part of theoretical neuroscience and computational neuroscience. Neural network models in artificial intelligence are usually referred to as artificial neural networks (ANNs) .These are essentially simple mathematical models defining a function or a distribution over or both and but sometimes models are also intimately associated with a particular learning algorithm or rule. A common use of the phrase ANN model really means the definition of a class of such functions where members of the class are obtained by varying parameters, connection weights, or specifics of the architecture such as the number of neurons or their connectivity.

\subsection{Welding:-}

Welding is a process of joining of two metals either same or different with the application of heat with pressure with or without filler rod. Use of welding in today's technology is used in every branch of industries, mechanical industries etc. Many different energy sources can be used for welding, including a gas flame, an electric arc, a laser, ,Ultrasound, an electron beam etc. While often an industrial process, welding can be performed in many different environments, including open air, under water and in outer space it can also be done in vacuum also. With the help of welding technology we can get strength up to $100 \%$. It is very easy to weld most of the material at any direction welding equipment can be transported at the work place easily.

\section{Welding Optimizations Techniques}

Now a day artificial neural network are widely used for optimization purpose so the purpose of our study is to analysis the process of welding using these techniques of soft computing .

Parikshit Dutta, Dilip Kumar Pratihar do Modelling of TIG welding process using conventional regression analysis and neural network-based approaches [1] and they conclude that conventional regression analysis was carried out based on full factorial design of experiments (DOE) and two neural 
network-based approaches (i.e., back-propagation algorithm and genetic-neural system) were developed and they compare both approach and they find that the neural network approach is more better than that conventional regression analysis[1] because Neural network based approach can carry out interpolation with in the a range. They find that back propagation neural network sows better performance compared to the genetic-neural system initially but after same time (near about 60,000 iteration ) second approach gives better performance It might be due to the fact that the solutions of BPNN were still lying on the local basin of the deviation (error) function, whereas the GA continued its search on a wider space .

M. Ericsson, R. Sandstrom perform an experiment in which they perform experiment on $\mathrm{Al}-\mathrm{Mg}-\mathrm{Si}$ alloy 6082 and compare the property of friction stir welding with MIG and TIG welding and they find that the fatigue strength of friction stir welding is higher than MIG and TIG welding for same material. TIG welded shows better fatigue performance than MIG, they find that mechanical and fatigue property of friction stir welding is independent of welding speed in the range of low to high commercial welding speed in this alloy. softening behaviour around FS weldments was good and a fair representation of the measured hardness profiles as a function of welding speed was found. how the gas influence the bead profile it is shown in the paper written by p.sethiya, abdul jaleel Sathiya P, Abdul Jaleel MY. Influence of shielding gas mixtures on bead profile and micro-structural characteristics of super austenitic stainless steel weldment by laser welding [3].

Rakesh Kumar, Ulrich Dilthey, D.K. Dwivedi, P.K. Ghosh say in his paper "Thin sheet welding of Al 6082 alloy by AC pulse-GMA and AC wave pulse-GMA welding" Materials and Design 30 (2009) 306-313 they conclude that by changing the EN ratio depth of penetration can be control if we increase the EN ratio a light or shallow penetration is achieves by which we can get a excellent welds of thin plate, they find that the mechanical property of the thin sheet were quite good tensile strength of the alloy which is taken for the study 6082 alloy weld is more than $200 \mathrm{MPA}$ and \% elongation is more than $2 \%$.

Kamal Pal say in his paper "Study of weld joint strength using sensor signals for various torch angles in pulsed MIG welding" he focus on the influence of the pulse parameters at various torch angles on the tensile Properties of low carbon steel butt weld in pulsed metal inert gas welding. The interface of weld zone and heat affected zone was found to be the weakest area due to significant variation of weld microstructure. He found that change in torch angle is significantly influence the weld bead characteristic and its microstructure. After performing the experiment he concludes the following point. area of weld joint, weld dilution, weld toe angle, bead width ratio and hardness variation strongly influenced the weld joint strength for all torch angles. arc sound kurtosis, arc power and weld peak temperature were found to be useful to monitor weld joint quality. However, these sensors' outputs are highly torch angle dependent.

Jean-Pierre Planckaert say in his paper "Modelling of MIG welding with experimental validation using an active contour algorithm applied on high speed movies" the design and testing of a physical model for short arc MIG/MAG welding was presented. Two approaches of hybrid model are used in this paper the arc state during which a drop forms at the end of the electrode, and a short-circuit state when the liquid drop transfers to the weld pool. It is shown that model predictions are consistent with experimental data in both Arc and shortcircuit states[6] .

Tetsumi yuri Effect of structure on high-cycle and low cycle fatigue properties MIG welded A5083 aluminium alloys at cryogenic temperature. It is fined in this in the highcycle fatigue test the S-N curves of A5083 base and A5183 weld metal shifted to higher stress level i.e the longer life side. At low test temperature the S-N curve of A5083 base metals are higher than those of A5183 weld metal at all test temperature. The ratio of FS to TS for A5183 weld metals was slightly lower than those of A5083 base metals at all test temperature. Although those of Austenitic stainless steel weld metals at $4 \mathrm{~K}$ were substantially decreased to about 0.4 [7] [8]. The effect of the blowholes on high-cycle fatigue properties are not clear or significant however fatigue crack initiation site in A5183 weld metals from blowholes if the blowholes are located near the specimen surface. Fatigue lives of A5183 weld metals were slightly shorter than those of A5083 base weld metals at cryogenic temperature. However the fatigue lives of A5183 weld metals at $4 \mathrm{~K}$ were superior to those of A5083 weld metal [8] .Giovanni Tani say in his paper of "The influence of shielding gas in hybrid LASER-MIG welding"

Sukhomay pal, Surjya k.pal, Arun k. samantaray say in his paper "Artificial neural network modelling of weld joint strength prediction of a pulsed metal inert gas welding process using arc signals" they monitor in pulsed metal inert gas welding process they developed a multilayer neural network to predict the ultimate tensile stress (UTS). They use 6 parameter which are pulse voltage, pulse frequency, background voltage, welding speed, and wire feed rate. They use root mean square (RMS) values of welding current and voltage are used as input variables and ultimate tensile stress is output. When they compare the welding strength given by the multiple regression to the result given by the Artificial Neural Network predicted result they found that ANN gives better result than the multiple regression result [10].

p..sathiya say in his paper of "optimization of laser welding process parameters for super austenitic stainless steel using artificial neural networks and genetic algorithm" he established a models are used for optimizing the process parameters using genetic algorithm (GA) and he optimize the solution for three different gases and their respective responses are obtained[1][4]. 
He investigate on the implementation of laser welding of similar joints is carries out and the relationship between the input parameters such as beam power, travel speed and focal position with the output parameters like tensile strength, depth of penetration and bead width is modeled through ANN. It is fined that ANN model is suitably integrated with optimization algorithm like GA to optimize the welding parameter [2] [5] .

For the optimized welding parameter of GA, the laser welding joints were processed. Joint exhibit better quality. The good agreement between the theoretically predicted (GA) and experimentally obtained tensile strength, depth of penetration and bead width confirm the application of process parameter in the welding process.

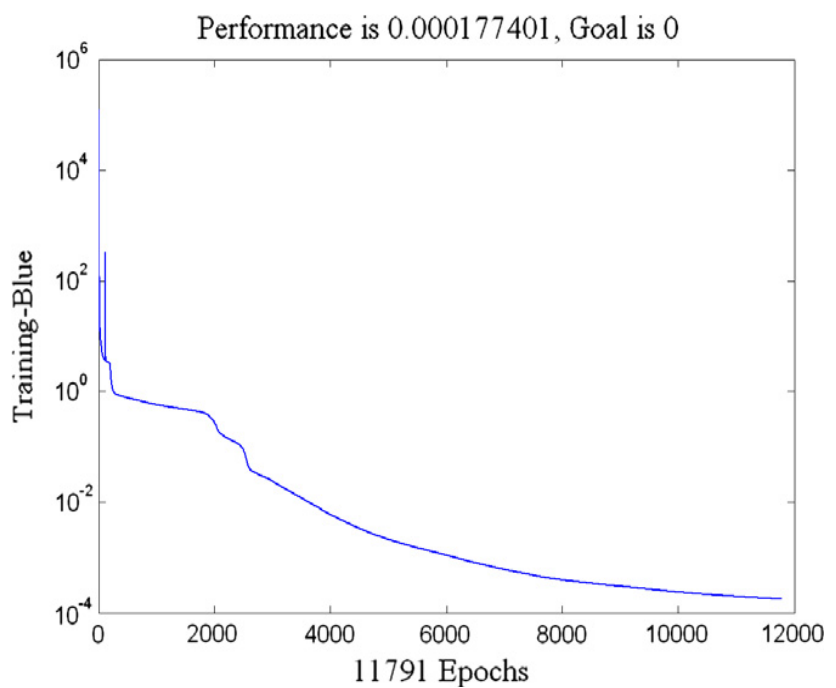

Figure 2. Graph representing mapping between percentage error and number of iteration for LBW with argon

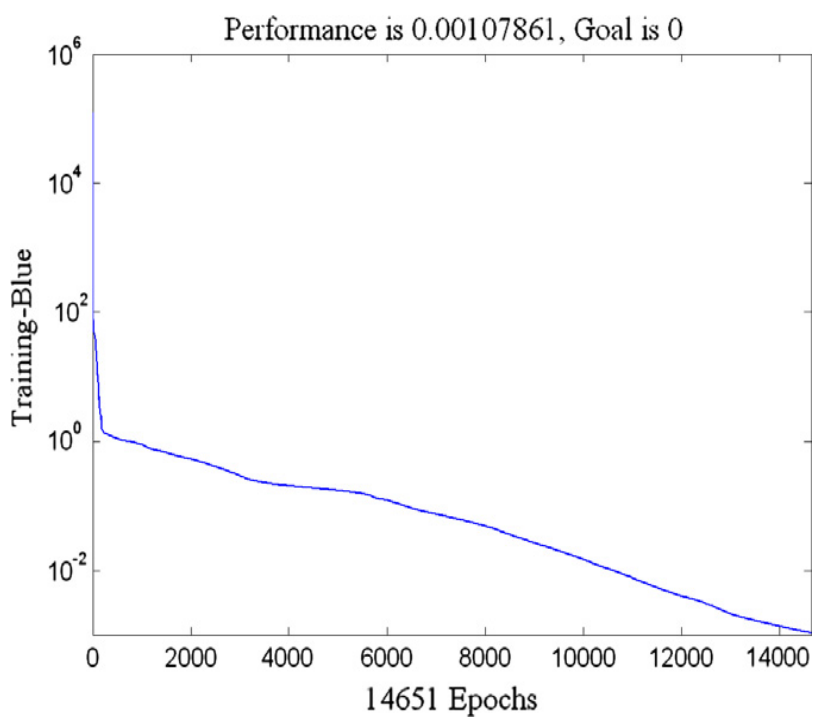

Figure 3. Graph representing mapping between percentage error and number of iteration for $\mathrm{LBW}$ with helium p.sathiya, K. Panneerselvam, R Soundarajan say in his paper of "optimization of laser butt welding process parameters using artificial neural networks and genetic algorithm for super austenitic steel" [14]in this paper same thing is done as shown above he take input parameter such as beam power, travel speed and focal position with the output parameters like tensile strength, depth of penetration and bead width is modeled through ANN. It is fined that ANN model is suitably integrated with optimization algorithm like GA to optimize the welding parameters. Similar experiment is done by the p.sathiya K. Panneerselvam, m.y. Abdul jaleel in this paper "optimal design for laser beam butt welding process parameter using artificial neural networks and genetic algorithm for super austenitic stainless steel"[15]

In this work laser beam welding was used for joining AISI $904 \mathrm{~L}$ super austenitic stainless steel with three shielding gases (argon, helium and nitrogen) In this work, the weld bead geometry such as depth of penetration (DP),bead width(BW) and tensile strength (TS) of the Joints madeofAISI904Lsuperaustenitic stainless steel were estimated. The model was developed to predict the depth of penetration (DP), bead width(BW) and tensile strength(TS) through Artificial Neural Networks technique for three different environments (Argon, Helium and Nitrogen). This type of model has been evaluated by means of the percentage deviation between the predicted values and the actual values. The developed ANN model is suitably integrated with optimization algorithms like GA to optimize the welding parameters. the laser welding joints were processed. Joints exhibit better quality. The result given by the ANN genetic algorithm GA and the result coming out from the experimental process is confirms the applicability for all the outputs for tensile strength, depth of penetration and bead width in welding process . 


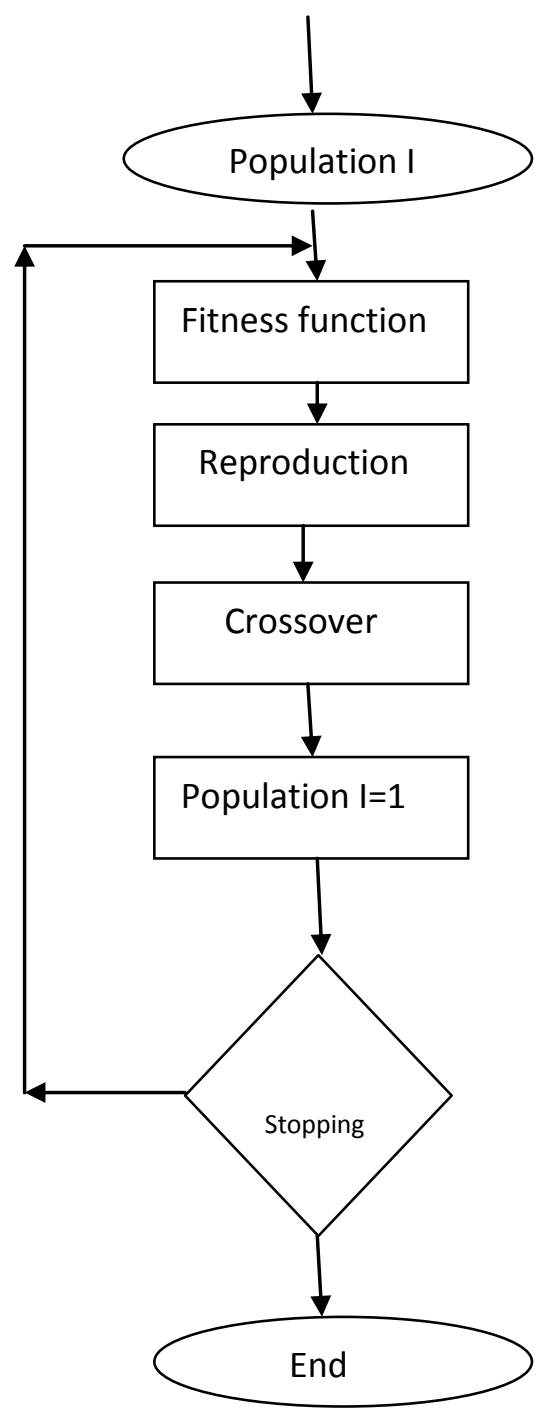

Figure 4. Genetic algorithm :Optimization process
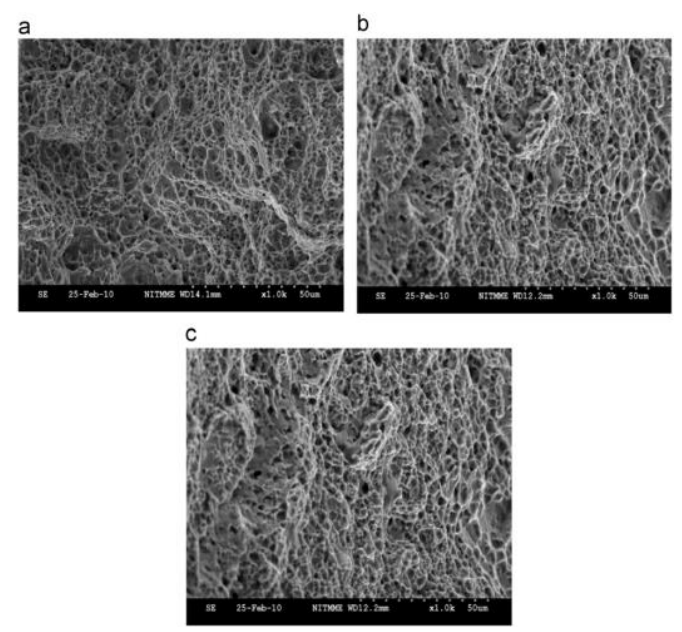

Figure 5. Complex SEM fractrograph microstructure $(\mathrm{a} \longrightarrow \mathrm{Ar}, \mathrm{b} \longrightarrow \mathrm{He}$ and $\mathrm{c} \longrightarrow \mathrm{N})$.

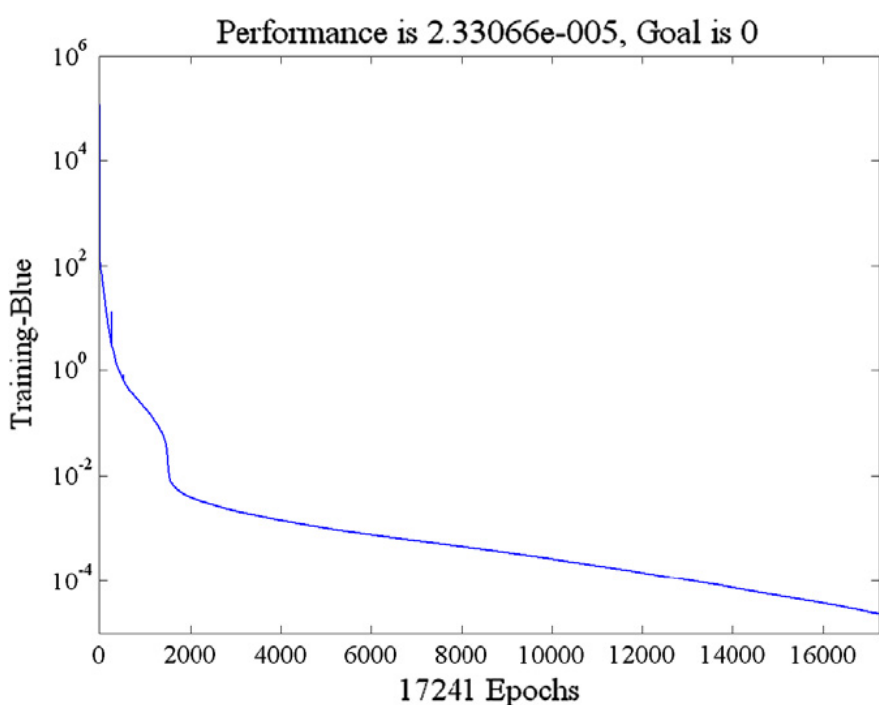

Figure 6. Graph for percentage errors and number of iterations for $\mathrm{LBW}$ with nitrogen

Rakesh malviya in his paper "Tuning of neural networks using particle swarm optimization to model MIG welding process"[13] he use the particle swarm optimization technique to model mig welding process ,in this paper four approaches have been developed and their performances are compared. First and second approach is deal with tuning of multi-layer feed forward neural network and Radial basis function neural network, respectively as shown in the figure .

Hakan Ates say in his paper "Prediction of gas metal arc welding parameters based on artificial neural networks" in this paper mr. Hakan Ates use Artificial neural network and predict for metal inert gas welding the output parameter tensile strength, elongation and weld metal hardness, impact strengthare investigated. Artificial neural network(ANN) is trained by deltabar-delta algorithm it is find that artificial neural network is gives better result error is between -1.238260870 and 3.340740741 is not so much high. So we can say that ANN can successfully integrated.[8] 
D.S. Nagesh, G.L. Datta say in his paper "Prediction of weld bead geometry and penetration in shielded metal-arc welding using artificial neural networks"[15] they investigate tha bead geometry and bead penetration. Experiment was conducted on grey cast iron welded with mild steel electrode It was observed that high arctravel rate and low arc-power normally produced poor fusion if work piece is preheated than it gives better result.as we increase in arc travel speed both bead width and bead height is decreases. If we keep arc length constant and increase electrode feed rate the penetration and HAZ increase. Bead width increase and bead height is decrease if we increase in arc-length keeping electrode feed rate constant. It is find that ANN gives accurate result.

In third and fourth approach a back propagation algorithm has been used with particle swarm optimization to tune radial basis function neural network. It is find that the performance of hybrid approaches (third and fourth) are found to be better than that of the other two.as we know that ANN is very useful tool which express the relationship between input and output multiple input and output can also be tackle with the help of ANN. In this regard, Feedforward neural networks (FFNNs) may be suitable to establish input-output relationships of a process. In FFNNs, information is passed into one direction, that is, starting from the input layer toward the output layer through the hidden layer. A few FFNNs, such as Multilayer feed-forward neural networks (MLFFNNs) and Radial basis function neural networks (RBFNNs) have been used to establish the said relationship. Radial basis function neural networks (RBFNNs) is better option for learning and adaption of technique because of its less computational complexity compare to (MLFFNNs). Both gives the same level of accuracy in prediction. The performance of RBFNN depends upon its structure decided by the number of its hidden neurons, parameters of radial basis functions used, connecting weights between the hidden and output layers of the networks and others. It is important to mention that various methods have been adopted to decide an appropriate number of hidden neurons for the RBFNNs. The number of hidden neurons of an RBFNN may also be kept equal to the number of clusters formed by the input-output data to be modelled. In Particle swarm optimization (PSO) is one of the population based evolutionary computing techniques, where the population is called swarm[11][12].

Input parameters in this welding technique is taken as welding speed, arc voltage wire feed rate gas flow rate nozzle-to-plate distance torch angle and output parameters ore bead height bead width bead penetration
Input layer Hidden layer Output layer

$[\mathrm{v}]$

$[w]$

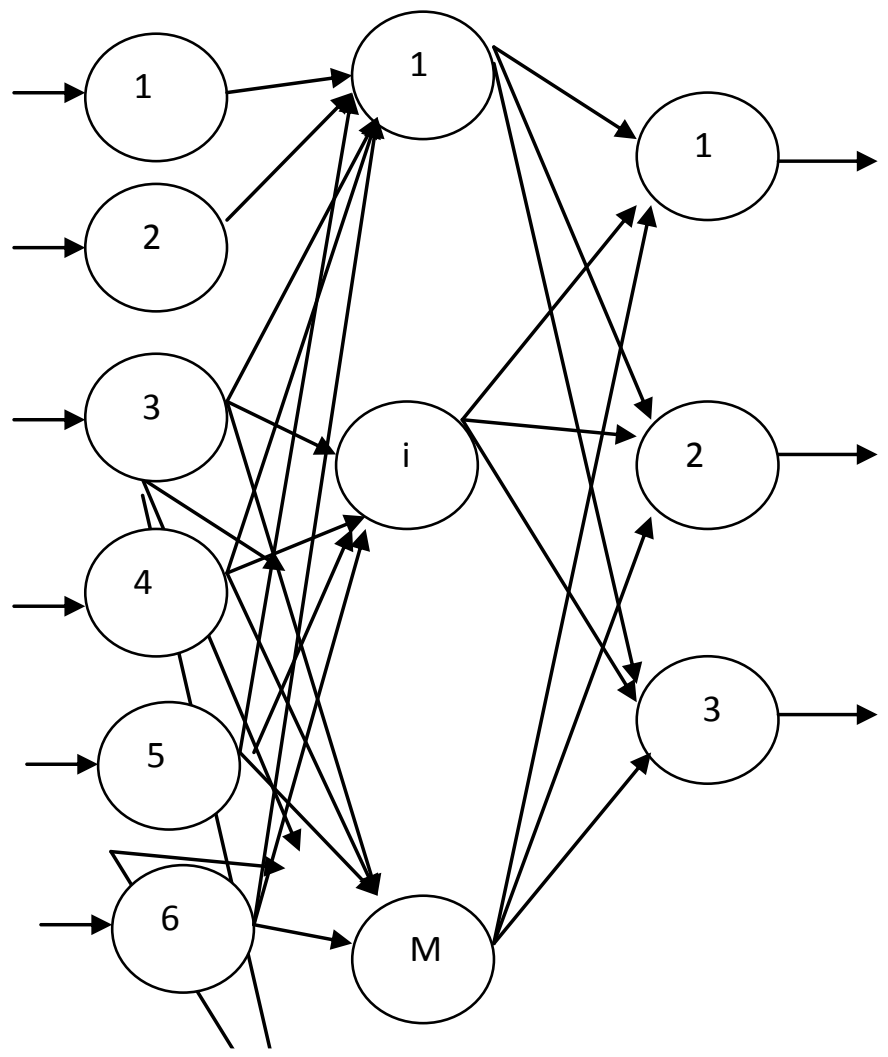

Linear T.F. Tan-sigmoid T.F.

Log-sigmoid T.F.

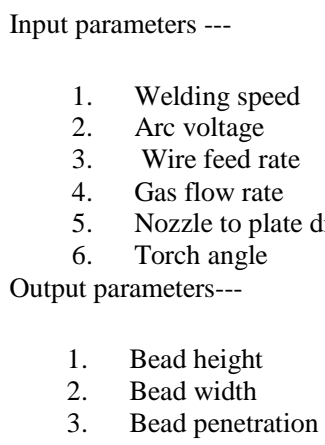

Figure7. A systematic view of MLFFNN 
Inputs

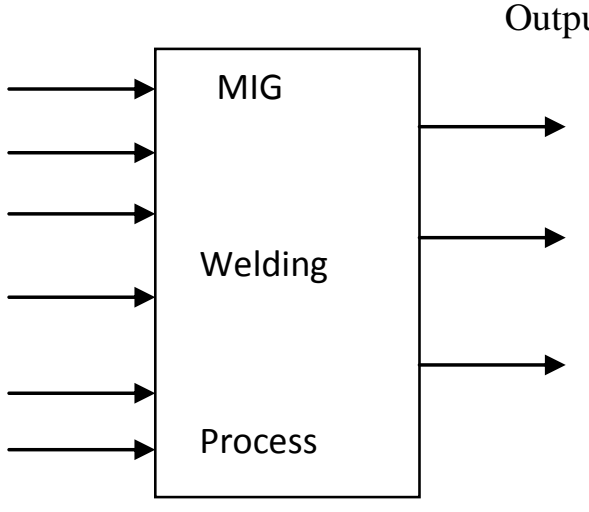

Inputs--

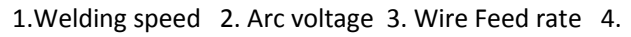

Gas flow rate 5 . Nozzle to plate distance 6 . Torch angl

Outputs---

1.Bead height 2. Bead width 3. Bead pentration

Figure 8. Input-output specifications of MIG Welding process.

PSO algorithms are similar to the genetic algorithm the PSO starts with the random initialization of population particles in the search space. The PSO algorithm works based on the social behaviour of particles in the swarm. Therefore, it finds the global best solution by simply adjusting the trajectory of each individual toward its own best location and toward the best particle of the entire swarm at each time step (generation).

\section{Conclusion}

Artificial neural network are not limited to the boundaries of computer science problems but it is widely used in different application of mechanical and agriculture domain . This paper includes a detailed study of utilization of artificial neural network in welding technology. Experimental analysis is given to present a clear picture of certain trends in welding that are taken in different cases .

\section{REFERENCES}

[1] Parikshik dutta, Dilip Kumar pratihar do modelling of TIF welding process using conventional regression analysis and neural network-based approaches. journal of Materials Processing Technology 184 (2007) 56-68

[2] P. Sathiya 爪, K. Panneerselvam, M.Y. Abdul Jaleel "Optimization of laser welding process parameters for super austenitic stainless steel using artificial neural networks and genetic algorithm" Materials and Design 36 (2012) 490-498

[3] Sathiya P, Abdul Jaleel MY. Influence of shielding gas mixtures on bead profile and microstructural characteristics of super austenitic stainless steel weldment by laser welding. International Journal of Advanced Manufacturing Technology 2011;54:525-35

[4] Sathiya P, Abdul Jaleel MY, Katherasan D, Shanmugarajan B. Optimization of laser butt welding parameters with multiple Performance characteristics. International Journal of Optics \& Laser Technology 2011;43(3):660-73.

[5] MATLAB neural network toolbox, The MathWorks Inc., Prentice- Hall, Engle- wood Cliffs; (1998).

[6] Jean-Pierre Planckaert El-Hadi Djermoune a,, David Brie $a$, Francis Briand b, Frédéric Richard Modeling of MIG/MAG welding with experimental validation using an active contour algorithm applied on high speed movie

[7] Tetsumi yuri "Effect of structure on high-cycle and low cycle fatigue properties MIG welded A5083 aluminium alloys at cryogenic temperature"

[8] Hakan Ates "Prediction of gas metal arc welding parameters based on artificial neural networks" Materials and Design 28 (2007) 2015-2023

[9] Kamal Pal, Surjya K. Pal Study of weld joint strength using sensor signals for various torch angles in pulsed MIG welding CIRP Journal of Manufacturing Science and Technology 3 (2010) 55-65

[10] sukhomay pal, Surjya k.pal, Arun k. samantaray "Artificial neural network modeling of weld joint strength prediction of a pulsed metal inert gaswelding process using arc signals" journal of materials processing technology 202 (2008) 464-474

[11] Rakesh malviya, Dilip pratihar "Tuning of neural networks using particle swarm optimization to model MIG welding process" swarm and evolutionary computation 1 (2011) 223-235

[12] Sathiya.p, K.Panneerselvam R Soundarajan"optimal design for laser beam butt welding process parameter using artificial neural networks and genetic algorithm for super austenitic stainless steel" optics \& technology 44 (2012) 1995-1914

[13] Neural Network Toolbox ${ }^{\mathrm{TM}}$ User's Guide R2012b Mark Hudson Beale, Martin T. Hagan, Howard B. Demuth

[14] Rakesh Kumar a, Ulrich Dilthey b, D.K. Dwivedi a,*, P.K. Ghosh c "Thin sheet welding of Al 6082 alloy by AC pulse-GMA and AC wave pulse-GMA welding" Materials and Design 30 (2009) 306-313

[15] D.S. Nagesh, G.L. Datta "Prediction of weld bead geometry and penetration in shielded metal-arc welding using artificial neural networks" Journal of Materials $\begin{array}{lllll}\text { Processing Technology } & 123 & \text { (2002) } & \text { 303-312 }\end{array}$ 